\title{
¿PODEMOS ESTIMAR EL IM PACTO DE LAS TRANSFORMACIONES DEL PAISAJE SOBRE LA ECODIVERSIDAD?
}

\author{
Juan J. NeIFF(1); Marcelo RoLóN (2) y Sylvina L. CASCO(1)
}

RESUMEN: Se han propuesto distintos criterios para medir la diversidad a nivel local (alfa y beta diversidad) a nivel regional (gama diversidad) y también a nivel del paisaje (ecodiversidad). De todas estas aproximaciones a la complejidad biótica en los ecosistemas, la ecodiversidad permite conocer la disponibilidad y la conectividad entre hábitat y lograr una idea de la variabilidad espacial de los ecosistemas. Se presentan siete índices de ecodiversidad y se discute sus ventajas y desventajas para el análisis de paisajes muy disturbados. Se analizó el paisaje del Establecimiento Las M arías, en el NE de Corrientes, que tiene algo más de 30.000 ha, comprendiendo 12.000 ha de sistemas forestales nativos y cultivados y 18.000 ha. dedicadas a té, yerba mate y policultivos. Se utilizó imágenes L andsat 7 y el procedimiento de Ecological L and Classification (ELC), para identificar las principales unidades de paisaje (bosques, pasturas, cuerpos de agua, diferentes cultivos). Se encontraron tres subsistemas de paisaje diferentes, se obtuvo información del número de polígonos y de la superficie comprendida en cada uso del paisaje y se la comparó cuantitativamente mediante varios índices. Se concluye que una determinada unidad de paisaje puede tener muy diferente diversidad, según el contexto de paisaje en el que se encuentre incluida.

ABSTRACT: Different approaches to measure the diversity at local level (alpha and beta diversity), at regional level (gamma-diversity) and also at the landscape level (ecodiversity, in sense of Naveh) have been proposed. Of all these approaches, the ecodiversity allows to know the habitat availability, the connectivity among habitat and to achieve an idea of the space variability of the ecosystems. Seven indexes of ecodiversity were tested and discusses their advantages and disadvantages for the analysis in a very disturbed area in the northeast of Corrientes. The results are presented for Las M arías Company that has something more than 30,000 hectares of those which 12,000 hectares are occupied with native and cultivated forest and 18,000 ha are devoted to tea, yerba mate, to the livestock breeding and aromatic plants. It were used satelital images $L$ andsat 7 analyzed with A RC V iew 3.2 and the procedure of Ecological Land Classification (ELC) to identify the main landscape units (forests, grasslands, water bodies). With this information three different subsystems of landscape were identified. The ecodiversity indexes were used to compare the ecodiversity patterns with the biotic complexity. We concludes that a certain landscape unit should be very different in specific di versity, according to the landscape context in which is included.

Palabras claves: biodiversidad, ecología de paisaje, ecodiversidad, fragmentación, índices de diversidad.

Key words: biodiversity, landscape ecology, ecodiversity, fragmentation measures, diversity index.

\section{INTRODUCCIÓN}

Desde la década del ' 60 se ha producido abundante literatura sobre evaluación ambiental, incluyendo procedimientos de identificación y calificación de impactos has-

(1) Centro de Ecología A plicada del Litoral (CECOAL-CONICET). Ruta 5 km 2,5 (3400) Corrientes. E-mail: neiff@arnet.com.ar; sylvina@arnet.com.ar (Pedido de Separatas)

(2) Establecimiento “Las Marías”, Virasoro, Corrientes. mrolon@asmarias.com.ar 
ta el uso de matrices y otros medios, a veces sofisticados, para cuantificar consecuencias de acciones antrópicas sobre el medio natural. La evaluación cuantitativa de estos impactos es siempre difícil, debido a que la percepción de al gún impacto es subjetiva y depende del momento en que sea percibido, del contexto socio-geográfico de la valoración, del estado previo de los ecosistemas y de otras causas que se modifican en forma dinámica (Etchechuri et al., 2002).

\section{INTRODUCCIÓN}

Desde la década del ' 60 se ha producido abundante literatura sobre evaluación ambiental, incluyendo procedimientos de identificación y calificación de impactos hasta el uso de matrices y otros medios, a veces sofisticados, para cuantificar consecuencias de acciones antrópicas sobre el medio natural. La evaluación cuantitativa de estos impactos es siempre difícil, debido a que la percepción de al gún impacto es subjetiva y depende del momento en que sea percibido, del contexto socio-geográfico de la valoración, del estado previo de los ecosistemas y de otras causas que se modifican en forma dinámica (Etchechuri et al., 2002).

U na preocupación de la mayoría de los análisis de impactos, es la modificación de la biodiversidad como consecuencia de las actividades humanas sobre los ecosistemas, si bien se tropieza con la falta de indicadores que permitan comparar el estado de la biodiversidad de un sistema en etapas sucesivas de un proyecto.

Otra dificultad radica en que los índices de diversidad disponibles, comparan la cantidad de especies de un sistema, o parte de él, con la abundancia de sus elementos (número de individuos, biomasa) como medida de complejidad local (? y ? diversidad) $y$, con menor frecuencia, se dispone de herramientas para el análisis de la diversidad regional (generalmente expresada por la diversidad ?).

Tampoco existe consenso respecto de la escala de análisis en que uno u otro índice, resulta más eficiente $y$, hasta qué punto existe complementariedad en los resultados que puedan obtenerse mediante su utilización cuando son aplicados en un mismo bioma. M ás aún, el término "biodiversidad" es habitualmente usado sin especificar a qué nivel de organización se hace referencia (genes, células, organismos, de poblaciones, de comunidades, de paisajes). En estas condiciones el análisis puede resultar confuso, poco útil (Solbrig, 1991, Hawksworth, 1995).

La biodiversidad de determinado sector de la biosfera depende en gran medida de las condiciones locales (relieve, suelos, microclimas) pero también, del contexto geográfico-ecológico y evolutivo en que ese predio se encuentra incluido (Solbrig, 1991). La presencia de paisajes adyacentes inalterados, de corredores ecológicos o la dirección de los flujos de información, pueden determinar que determinado paisaje tenga distinta diversidad según la posición que ocupe en una cuenca hidrográfica (N eiff, 2001).

Cuando se trabaja en la evaluación ambiental a nivel de predios (1000 a 100.000

ha) el análisis de biodiversidad de paisaje resulta una herramienta útil porque permite utilizar distintas escalas y valorar factores de contexto, logrando una perspectiva bio regional (WRI-UICN/PNUMA, 1992). 
El ensamble de organismos, su complejidad, distribución y abundancia, depende principalmente de la configuración del paisaje a escala regional, sub-regional y local (Naveh, 1994; M cGarigal y Marks, 1995; M iller, 1996). Los procesos de sustitución y/o de empobrecimiento del paisaje natural conducen a la fragmentación, proceso caracterizado por la reducción del área total ocupada por el paisaje prístino y por la subdivisión del mismo en parcelas de menor tamaño. El tamaño, forma y distancia entre las parcelas tiene mucha importancia para explicar los cambios en la biodiversidad (Zunino, 1986, M cGarigal, 1995; M iller, 1996; TNC, 2000).

En esta contribución se presentan algunos índices que pueden utilizarse para el análisis de los impactos sobre la ecodiversidad, es decir, la diversidad a nivel del paisaje (Naveh, 1994). Para probar los índices propuestos se analizó el conjunto de predios del NE de Corrientes pertenecientes al Grupo Las M arías.

\section{MATERIALES Y METODOS}

Área de estudio. Se analizó el Establecimiento $L$ as M arías S.A., perteneciente al Grupo $L$ as $M$ arías, situado en la localidad de Gobernador $V$ irasoro, nordeste de Corrientes (28066' 42" lat. S; 56903'20" long. O, Fig. 1). Los predios se encuentran en una zona en que los ecosistemas han sido al terados en más de ochenta por ciento de la superficie por actividades agropecuarias y forestales desde fines del siglo XIX.
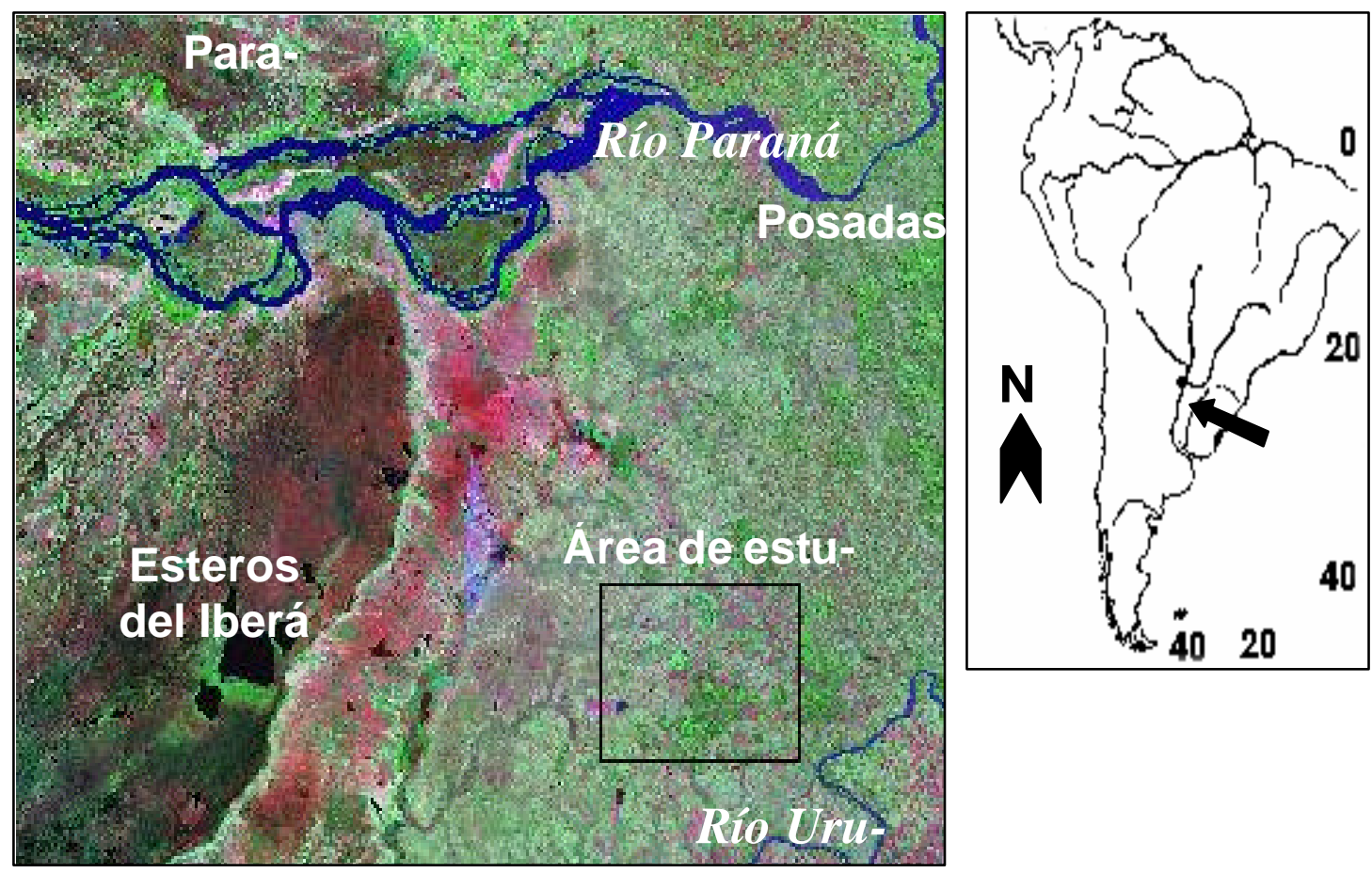

Fig. 1: Ubicación del área de estudio 
Corresponde al ambiente de colinas bajas, típicas del sur de la provincia de Misiones, denominadas "Colinas y Llanuras onduladas del noreste" (Capurro y Carnevali, 1985), con pendiente suave, depresiones someras y suelos rojos, predominantemente arcillosos. Tiene clima húmedo con frecuentes excesos hídricos en otoño-primavera y poca deficiencia de agua, principalmente en verano.

EI Establecimiento inició su actividad con la producción ganadera en la segunda década del siglo XX y posteriormente desarrolló plantaciones de yerba mate y de té. $L a$ actividad forestal comenzó a fines de la década de 1960, con la plantación de 500 ha de pino y eucalipto. Algunas áreas con bosque natural se conservaron, aunque aprovechando la madera de mejor calidad presente en los mismos. L as plantaciones forestales fueron establecidas en campos anteriormente dedicados a la producción de yerba mate o pastizales abandonados de antiguas prácticas ganaderas. Las sucesivas plantaciones fueron logradas mediante la optimización del uso de los recursos, ubicando a cada especie forestal en las mejores condiciones de suelo.

En las últimas décadas se ensayaron muy variadas formas de utilización de los predios, constituyendo finalmente un agrosistema de uso múltiple, con policultivos que conservan sectores de paisajes nativos que están siendo restaurados.

El Grupo L as M arías está integrado por varios establecimientos: Las M arías, Cerro V erde, Orsetti, A guaí, L eiva, B obby, Tres Capones, M aría Concepción, Tubomet, Y ohasá 1; Y ohasá 2; Y ohasá 3; Y ohasá 4; Y ohasá 5; Y ohasá V iejo; R oberts; La Península; La Victoria, San Justo, San Patricio, Santa Inés, El Playado, El Carmelo, A grocom, Garruchos, Singer, López, Calzada de Piedra, La M erced (de Las M arías) y La Merced que totalizan unas 30.000 ha.

En conjunto tienen 13.200 hectáreas de bosques nativos y plantaciones de Pinus elliottii, P. taeda y Eucalyptus grandis, que se destinan, principalmente, a la producción de madera aserrada, madera laminada y madera triturable. Se dispone además de numerosos ensayos de, al menos, una docena de especies forestales, en parcelas de tamaño variable y de combinaciones de éstas con pasturas implantadas, con cría de ganado, con yerba mate y té; cultivos de plantas aromáticas y una amplia gama de usos del paisaje, lo que determina un mosaico de parcelas de distintos tamaños y formas.

Procedimiento. Para identificar unidades de paisaje, se utilizó la Clasificación Ecológica de Tierras - ELC - (FEA RO, 1978), que es un sistema de clasificación que permite jerarquizar la importancia de cada sector del paisaje. Esencialmente, se basa en los principios de la ecología que consideran las relaciones temporales y espaciales entre los cinco componentes principales del paisaje: terreno, hidrología, clima, suelo y vegetación. Se digitalizó y procesó en A rc-V iew 3.2 la imagen satelital Landsat 7 TM de L as M arías (de febrero de 2000) para obtener algunos índices de la estructura del paisaje siguiendo los lineamientos propuestos por M cGarigal y M arks (1995), Naveh (1994) y O’Neill et al. (1999):

\& El número de parcelas de cada tipo de uso de la tierra y su relación respecto del total de usos.

\& El tamaño de las parcelas ("manchas", "parches" o "Iomas") en relación al área total.

- La forma de los parches (parcelas).

\& El desarrollo de la línea de borde de cada parcela. 
\& Valor medio (forma, área o número de parcela) para cada uso y la variación dentro de la estructura.

Todos los predios fueron considerados como un solo sistema a los fines de este análisis. Sin embargo, se separaron tres subsistemas ecológico-productivos: Tres Capones, Y ohasá 5 y Tubomet, que han sido sometidos a distintos sistemas de aprovechamiento en las últimas décadas y que tienen diferencias estructurales (en la proporción, tamaño, forma de las parcelas) y ecológicas (en la oferta de hábitat y en la conectividad entre las parcelas de una misma unidad de paisaje).

Se utilizaron 7 índices de organización del paisaje:

\title{
Indice No 1: R elación perímetro/radio del parche promedio
}

\author{
Perímetro de c/parche de uso \\ Superficie de c/parche de uso
}

Este índice es útil para establecer la posibilidad que posee determinado organismo o población de cubrir uno o más parches del patrón de paisaje, en tanto se conozca el radio de desplazamiento habitual de los animales. Se denomina también índice de forma. L os valores más bajos indican que los parches tienen forma circular. En algunos casos, los paisajes culturales crean extensos bordes (aumentan el perímetro) y modifican la conectividad entre parches, con lo cual, se modifica la posibilidad de dispersión de los organismos. Puede suceder que al gunas poblaciones queden más expuestas a los predadores. El aumento del perímetro de las parcelas, en oportunidades, puede favorecer a las especies que viven en los bordes, en detrimento de aquellas que moran en los interiores de hábitat.

\section{Indice $\mathrm{N} 0$ 2: Porcentaje de importanc ia 1}

\author{
Superficie de cada uso $\quad \times 100$ \\ Superficie total de todos los usos
}

Proporciona una idea de la disponibilidad espacial (superficie ocupada) de los distintos usos del paisaje (Ej. área total de té respecto del área total de los usos) como recurso de hábitat para aquellos organismos con mayor posibilidad de seleccionar el hábitat, como por ejemplo, las aves.

\section{Indice $\mathbf{N}$ 0 3: Porcentaje de importancia 2}

\section{№ de poligonal es de cada uso $\times 100$ $\mathrm{N}$ - total de poligonales}

Idem que el anterior, sólo que para el cálculo utiliza el número de elementos homólogos (poligonales) que se encuentran en la imagen y los relaciona con el número total de poligonales de la misma. En cierta medida, es un indicador de la frecuencia con que determinado organismo podría encontrar ese tipo de parcela (Ej. té).

\section{Indice N 0 4: E codiversidad}

L a mayor riqueza faunística se relaciona generalmente con la mayor heteroge neidad del patrón de paisaje (Picket y W hite, 1985, W iens, 1996, Huston, 1999). Cuan- 
do la estructura del paisaje integra parcelas de diferentes usos, con otras de paisajes nativos remanentes de tamaño y forma variada, puede encontrarse una mayor of erta de hábitat para los organismos (fauna y flora) dado que se genera una multiplicidad de ambientes con distintos microclimas y balance de la energía.

$$
\text { Indice } N \text { - } 4_{1} \quad \mathrm{~d}_{1} ? \frac{\mathrm{S} ? \mathrm{1}}{\log \mathrm{N}}
$$

donde: $\mathrm{S}=\mathrm{N}$ ㅇ total de usos

$\mathrm{N}=$ suma de las superficies de las poligonales para todos los usos

Este índice asume que cada parcela (Ej. loma de té) es equivalente a una especie. Este índice fue desarrollado por M argalef como índice sintético que expresa la importancia den especies respecto del valor de importancia de todas las especies en la muestra. Si bien no ha sido utilizado para el análisis en la escala de paisaje, el rango de valores presentes en las imágenes, puede ser usado ventajosamente para representar sintéticamente la ecodiversidad de cada muestra anal izada. El Índice $4_{1}$ utiliza como parámetro de calidad a los usos (té, yerba, pinos) y como parámetro de cantidad, a la superficie que ocupa cada uno de ellos.

$$
\text { Indice } \mathrm{N}_{\mathbf{2}} \quad \mathrm{d}_{1} ? \frac{\mathrm{S} ? 1}{\log \mathrm{N}}
$$

donde: $\mathrm{S}=\mathrm{N}$ total de usos

$\mathrm{N}=$ suma de las poligonales para todos los usos

I dem que el anterior, sólo que utiliza como indicador de cantidad al número de poligonales de cada uso, en la muestra.

\section{Indice No 5: Tamaño de la parcela (loma) más grande}

En algunos casos, un paisaje estructurado por pocas células de gran tamaño tendría menor variación de hábitat, especialmente si se trata de cultivos. Esto haría esperable una mayor riqueza de especies.

Indice No5: Superficie de la parcela más grande para todos los usos

Este descriptor da una tendencia respecto del parcelamiento del paisaje y de la preponderancia que imponen las parcelas (parches) más grandes en el patrón de paisaje que se analiza.

Indice No52: Superficie de la parcela más grande de cada uso

Permite conocer la preponderancia espacial de al guno de los usos del paisaje (Ej. yerbales) en el contexto del paisaje. En el caso extremo, en el cual un uso (o unidad de paisaje) formara una única parcela, habría que esperar, además de un patrón espacial y temporal menos variable, menores posibilidades de conectividad con otros paisajes para aquellos animales que tienen un radio de desplazamiento corto. 


\section{Indice 6: C oeficiente de variación de las parcelas para cada uso (\%)}

Es uno de los indicadores sintéticos de mayor interés (índice compuesto por el valor medio de un atributo y por su variabilidad), debido a que proporciona mucha información sobre la organización y funcionamiento del paisaje. En algunos paisajes nativos en los que el coeficiente de variación (CV) es bajo, se reconoce una gran homogeneidad del medio biofísico, lo que determina que todos los sectores del patrón de paisaje tengan capacidad de soporte semejante.

L os coeficientes de variación al tos en el patrón de paisaje indican, generalmente, ventajas para la vida silvestre, especialmente para aquellos animales de menor radio de desplazamiento y para los que se benefician con la exploración de diferentes hábitat.

$$
\frac{\text { Desviación standard de la superficie de las lomas }}{\text { Promedio de la superficie de las lomas }} \times 100
$$

\section{Indice 7: Desviación standard del promedio del coeficiente de variación de todos los usos}

Este índice expresa la variabilidad general del sistema analizado y es de interés cuando las diferencias espaciales en el paisaje se relacionan con diferentes prácticas de manejo o de uso.

\section{RESULTADOS}

L os subsistemas, definidos en base a las condiciones actuales del paisaje y a las clases de uso, han sido representados en las Figuras 2 (clases de paisaje) y 3 (usos del suelo).

El subsistema Tres Capones se caracteriza por tener la mayor superficie ocupada por pastizales, áreas de manejo ganadero y por la existencia de la mayor superficie de monte nativo. El patrón de paisaje se completa con arbustales foliosos cultivados (yerbales de baja densidad y teales de densidad media) que están siendo convertidos en policultivos, con la incorporación de un estrato arbóreo de baja densidad (kiri, pino, eucalipto, etc).

L os humedales ocupan poca superficie aunque tienen mucha significación para la vida silvestre, dado que hay poblaciones de fauna que los habitan en forma temporal 0 permanente. L a mayor superficie de este subsistema se encuentra como un bloque, conformado por los establecimientos Tres Capones y L as M arías (más del $70 \%$ de la superficie total).

Por constituir la parte más antigua de L as M arías, el diseño de las parcelas refleja los criterios vigentes hasta hace pocas décadas: parcelas geométricas, de tamaño semejante, sin vías (senderos, corredores) que interconecten paisajes homólogos (parcelas de pasturas, 0 áreas de montes nativos entre sí, por ejemplo).

L os resultados de los parámetros cuantitativos analizados, son coherentes con lo descrito: valores de ecodiversidad medios a altos (Índices $4_{1}$ y $4_{2}$ en la Tabla 1 ) y los coeficientes de variación (Índice 7) también al tos.

En el subsistema Y ohasá 5 queda comprendida la mayor área de producción de té y de yerba diagramada con modernos criterios tecnológicos: alta densidad, mayor fre- 
cuencia de cosecha, corte bajo, mayor utilización de agroquímicos. El patrón de paisaje se completa con plantaciones de pinos y, en menor superficie, cultivos de eucaliptos. Las áreas ocupadas por pastizales son puntuales al igual que las áreas de bañados y de pajonales. V arios arroyos atraviesan los campos de la zona: de ellos, el Ibirá Ocai y el Y ohasá, forman planicies inundables (bañados y esteros de recarga).

El tamaño y la forma de cada loma son muy variables (Tabla 1) predominando las de forma irregular y de menor tamaño, intercaladas por cortinas (fajas) de eucal iptos 0 de pinos. L os tamaños de las parcelas de utilización son, en general, menores que en el subsistema Tres Capones, excepto en los bloques correspondientes a los establecimientos Cerro V erde, Orsetti y San Patricio. 


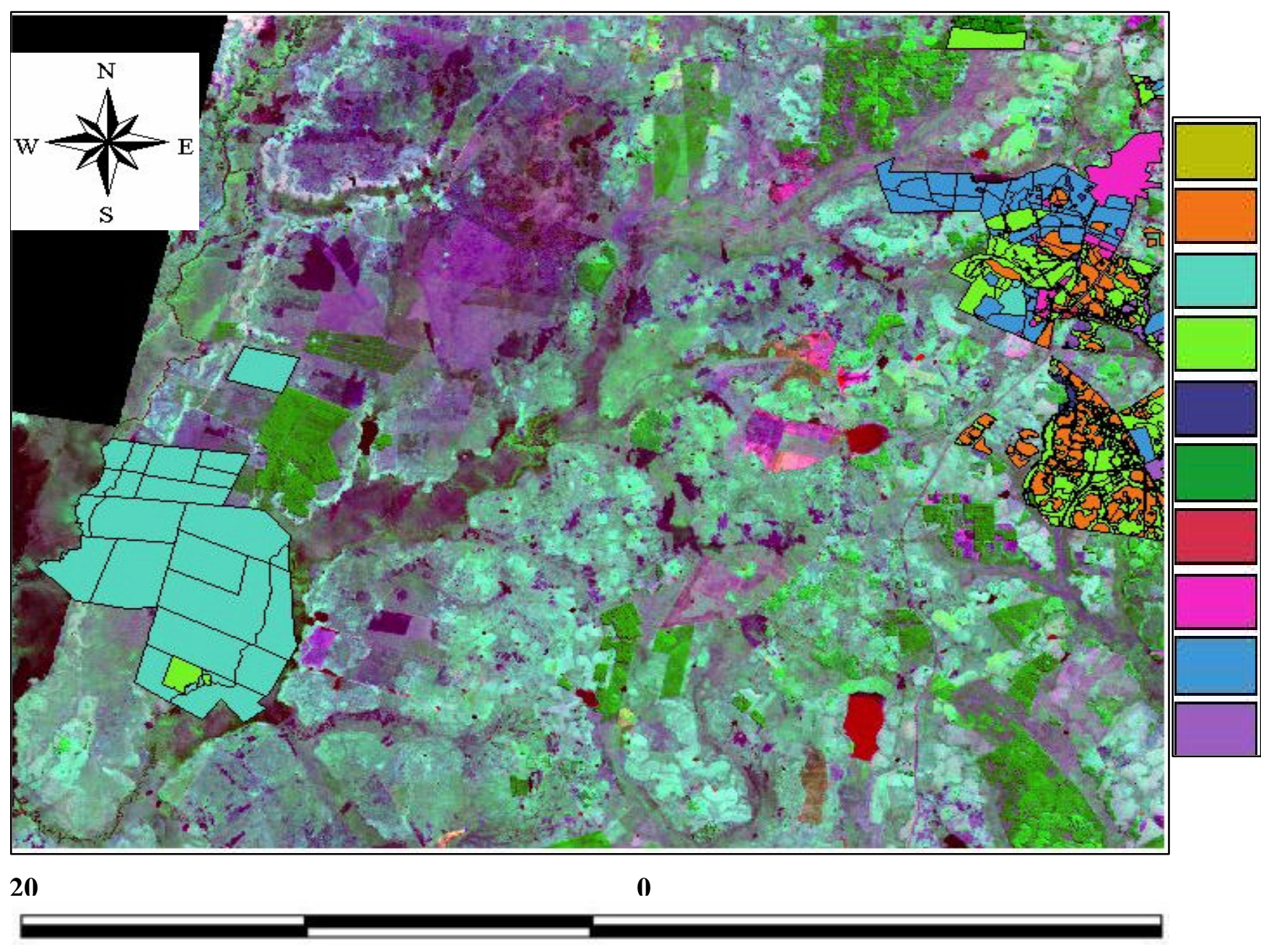

Aguas

Arbustales foliosos

Bañados

Bosques implantados

Bosques nativos higrófilos

\section{Esteros}

Misceláneas

Núcleos urbanos

Pastizales

Tala rasa

Fig. 2: Imagen del área estudiada con las clases de paisaje identificadas. 


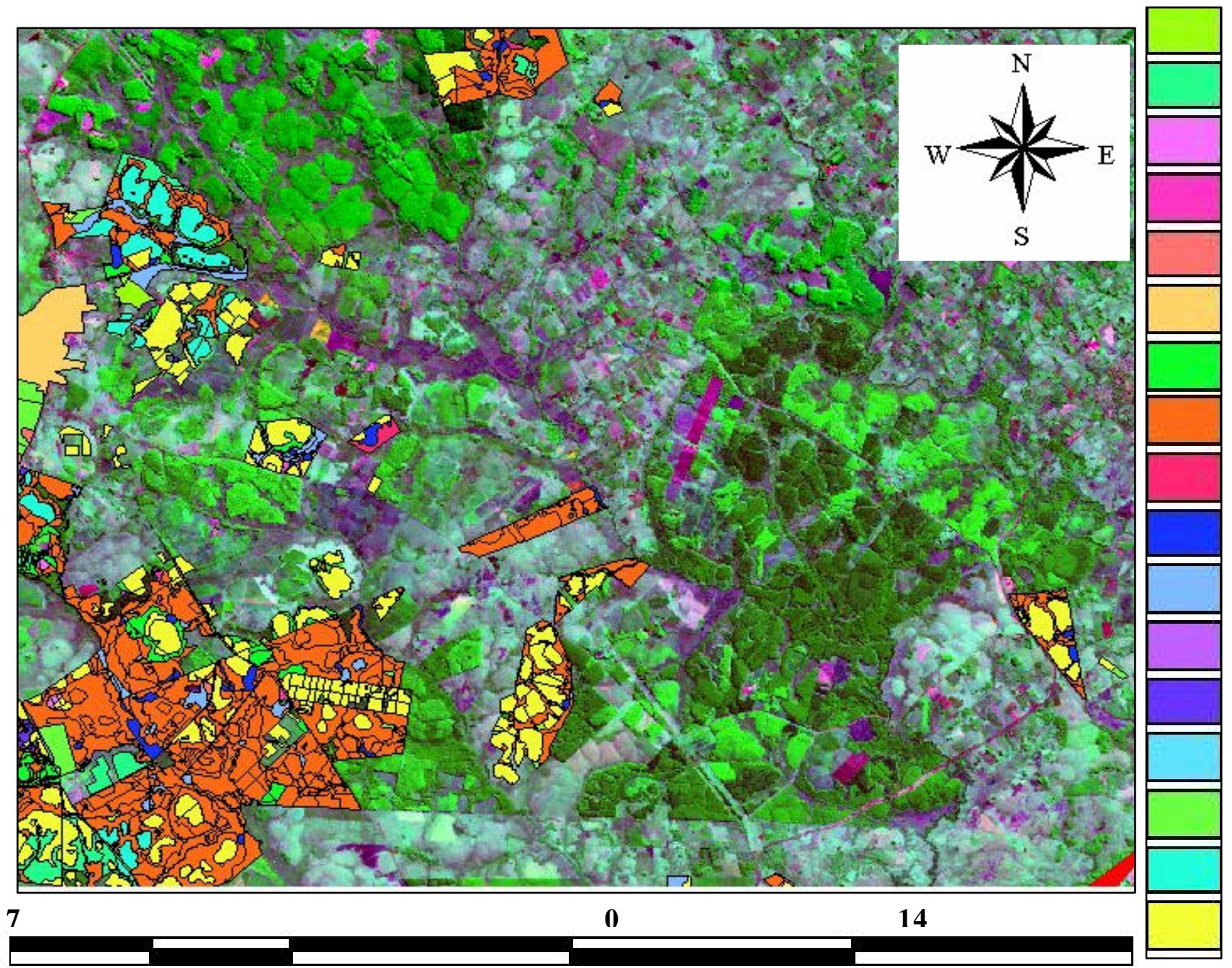

Fig. 3: Uso actual del paisaje en el área estudiada.
Aguas quietas

Areas agrosilvopastoriles

Aromáticas o medicinales

Capueras o capuerones

Caseríos, talleres, oficinas

Ciudades, poblaciones, villas, barrios

Fores taciones de Eucalyptus

Forestaciones de Pinus

Hierbas altas y tiernas

Isletas o capones

Pajonales

Palmera real

Plantaciones experimentales

Praderas cultivadas

Praderas naturales

Té

Yerba mate 
Hay buena conectividad entre las lomas y ecodiversidad alta, debida a la presencia de varias formas de uso y a parcelas de muy distinto tamaño y forma. Los teales y yerbales de alta densidad son manejados para lograr la mayor cobertura monoespecífica de la canopia, con prácticas mecánicas y con ayuda de productos fitosanitarios. No es extraño entonces que la fauna, en general, sea poco diversa y poco abundante.

El subsistema Tubomet presenta un patrón de paisaje con dominancia de forestaciones de pinos y de eucaliptos. Quedan incluidos dos bloques de diferente tamaño y forma. El bloque principal está formado por los establecimientos San J usto, Calzada de Piedra, Tubomet (ecositio más característico del subsistema), El Carmelo, Y ohasá 1, El Playado y López.

En este bloque las lomas con plantaciones recientes tienen forma y tamaño irregular contrastando con las más antiguas, donde se observa el diseño de damero de los yerbales y teales. El resto del bloque Tubomet tiene lomas, en general, pequeñas, irregulares, interconectadas a los caminos principales, que tienen disposición radial, lo cual favorece la conectividad entre parcelas.

El segundo bloque está constituido por el establecimiento Roberts que es una franja de terreno de $4,5 \mathrm{~km}$ de longitud por unos $0,6 \mathrm{~km}$ de ancho, situada entre la ruta provincial 68 y el arroyo Garabí. Por su forma tiene un extenso borde de contacto con campos dedicados fundamental mente a la ganadería extensiva. Los caminos internos funcionan como corredores entre el arroyo Garabí y las lomas forestadas.

El tamaño y la forma de las lomas es semejante al subsistema Y ohasá y la diferencia principal es que dominan el paisaje las forestas de pino y, con menor cobertura, las de eucal ipto, de distinta edad y de varias especies. Los teales y yerbales quedan incluidos entre los rodales de pino y de eucalipto. L os campos con tala rasa son más importantes que en los otros subsistemas. El arroyo Y ohasá atraviesa varios campos de este subsistema, funcionando como corredor natural.

Entre las peculiaridades del subsistema Tubomet, se encuentran varios sitios de arbustales en activa sucesión secundaria (capueras), bajos con claros y con bañados, forestas de baja productividad, que han sido colonizadas por el monte nativo hidrófilo y una amplia gama de rodales de diferente edad, tamaño, espaciamiento y especies.

Se identificaron diez clases de paisaje, quedando comprendidas en las mismas, al menos diecisiete unidades estructural y funcionalmente distintas como se esquematiza en la Figura 4 y que pueden definirse en las imág enes satelitales (Figura 2). Seis de estas unidades pertenecen a ambientes acuáticos y palustres, en tanto las restantes pueden considerarse como "ambientes de tierra firme". De estas últimas, dos corresponden a distintos tipos de praderas y dos a bosques implantados de pinos y eucalipto. 


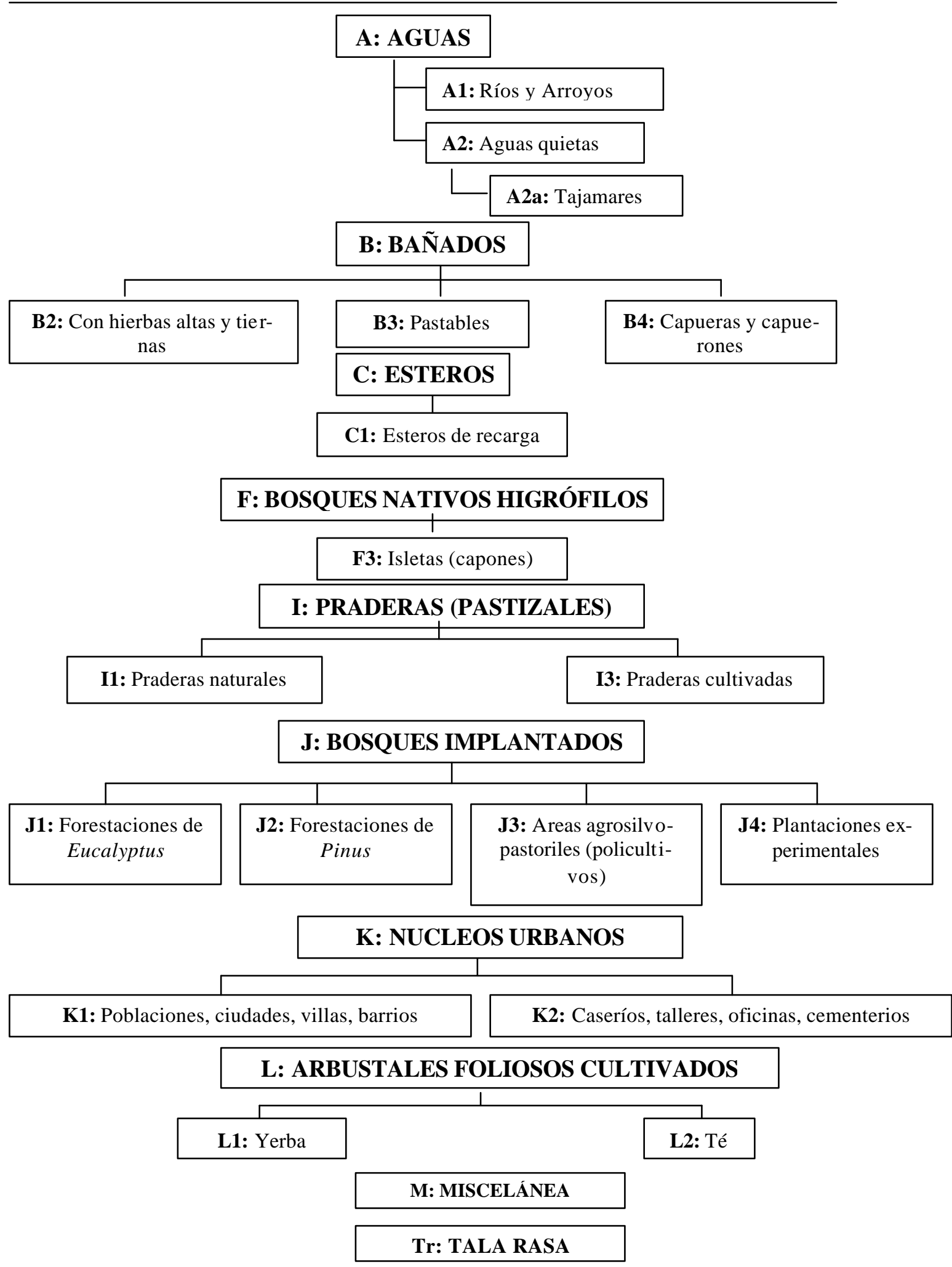

Fig. 4: Clases de paisaje identificadas en Las Marías. Los términos utilizados se encuentran en Neiff et al (2003). 
Los resultados de los índices fueron coherentes e indic aron una gran variación interna a nivel de cada uno y de todos los establecimientos analizados. Pudieron establecerse cuatro complejos de paisaje:

Patrón de paisaje tipo uno: la ecodiversidad (Indices $44_{1}$ y 4 en la Tabla 1 ) tuvo valores muy al tos en $Y$ ohasá 5 y en $Y$ ohasá $V$ iejo, como resultado de una gran variedad de usos del espacio en relación al tamaño de los predios. El Indice 2 acusó valores muy bajos, Io que indica una participación muy proporcional de los distintos usos del paisaje en cada uno de los establecimientos nombrados. En Y ohasá V iejo las plantaciones de té alcanzan al $42 \%$ de la superficie, encontrándose el resto distribuido en 25 usos diferentes. U na situación semejante se da en Y ohasá 5 que tiene 25 usos diferentes del suelo, de los cuales los yerbales de alta densidad ocupan más del $40 \%$ y los restantes usos tienen valores muy bajos en el Indice 2 .

El coeficiente de variación por usos (Índice 6) y el coeficiente de variación total (Indice 7) fueron igualmente altos con val ores próximos o superiores al $50 \%$. Se advierte que, para ambos establecimientos, el coeficiente de variación encontrado para los usos del paisaje y el coeficiente de variación total son muy semejantes. Es decir, el valor medio de la variación entre las lomas de cada establecimiento es semejante al desvío de este valor. Como corolario: muchos usos diferentes, en muchas parcelas, de tamaños muy variados.

Patrón de paisaje tipo dos: corresponde a los establecimientos Tubomet, López, Calzada de Piedra, San J usto y L a M erced. A juzgar por los resultados de los Indices $4_{1}, 4,6$ y 7, la ecodiversidad del paisaje es mucho menor que en el caso anterior, sin embargo el patrón de paisaje resulta muy heterogéneo por la gran diferencia en el tamaño de las lomas (parcelas) que integran el patrón. Los Indices 6 y 7 tienen coeficientes de variación muy próximos al 50\%. De los 13 usos principales del paisaje, 8 son forestales. Los distintos tipos de forestaciones ocupan el 86\% del patrón de paisaje (Indice 2 ).

Con este mismo patrón, EI Carmelo tiene 14 usos principales, de los cuales 10 corresponden a formaciones leñosas, que ocupan en conjunto el $77 \%$ del campo. Tanto Tubomet como El Carmelo son situaciones típicas del Subsistema Tubomet.

Patrón de paisaje tipo tres: los establecimientos M aría Co ncepción, R oberts y La Península, tienen muy baja ecodiversidad y coeficientes de variación próximos al $40 \%$. Hay parcelas de gran tamaño como en M aría Concepción, donde la parcela mayor tiene 1112 ha (Indice $5_{1}$ ), constituyendo la situación menos favorable para la vida silvestre.

En el caso de María Concepción, se encuentran extensos humedales (bañados, pajonales) ubicados en el interfluvio de los $\mathrm{A}$ A A yuí y A guapeí. L os valores de diversidad de fauna para pajonales son bajos en comparación con los que corresponden a montes nativos, por ejemplo. Sin embargo, serían esperables valores al tos si se compara la fauna de estos pajonales (ubicados en un corredor entre ambos arroyos) respecto de otra situación en la que los pajonales conformaran células aisladas.

En igual sentido, el establecimiento Roberts, con ecodiversidad y coeficiente de variación bajo, tiene una situación muy favorable por la forma del campo y por el contexto de paisaje en que se encuentra. 
L o expresado lleva a plantear que los valores de alfa diversidad dependerán en gran medida de un factor de contexto que debe ser evaluado para conocer la validez de los valores locales de diversidad.

Patrón de paisaje tipo 4: T res Capones constituye un patrón de paisaje muy diferente del resto del G rupo Las M arías en razón de ser, por su organización estructural y por su contexto, lo más próximo a la situación existente a comienzos del siglo pasado, sin desconocer las transformaciones ocurridas desde entonces en cada unidad de paisaje.

En el Establecimiento Tres Capones, la ecodiversidad puede considerarse media a baja (Índices 4 y $4_{2}$ ), con parcelas grandes (Índice $5_{1}$ ) dado que la mayor parte del paisaje corresponde a áreas de pastizal es de manejo pastoril, al gunos de los cuales están siendo convertidos en forestaciones. Hay parcelas de policultivos pero ocupan poca superficie al considerar todos los predios del establecimiento. En general predominan las parcelas grandes, aunque hay un número equivalente de parcelas pequeñas, lo que da coeficientes de variación del paisaje próximos al 60\%. Otra característica de este patrón de paisaje está dada por el factor de contexto que brinda una situación muy favorable para la ecodiversidad. Todo el límite del predio se relaciona con paisajes muy diferentes, incluyendo las áreas periurbanas de Gdor. V irasoro, la planicie de inundación del $\mathrm{A}$ A yuí, por el norte, con fisonomías de bañados, pajonales, pastizales y bosques fluviales, yerbales de baja densidad, bosques talados (talas rasas), ripieras, montes higrófilos nativos en capones próximos al $A$ ㅇ Santa M aría y los bañados de este arroyo, además de pequeños tajamares.

Considerando todas las zonas, en el pastizal de EI Playado se da el máximo valor del Índice 1 (16,23), lo que indicaría que la forma de la parcela de pasturas es la más irregular de todas. Es decir, que el borde de contacto con los paisajes vecinos es mayor y que la fauna (aún la de menor desplazamiento) puede contactarse con los paisajes vecinos. $V$ al ores menores se obtuvieron para Y ohasá 5, Y ohasá 2, 3 y 4; San J usto y Tres Capones. El cultivo de yerba mate de al ta densidad de EI Carmelo es el uso cuya forma se asemeja más a un círculo $(0,96)$. 
Tabla 1. Análisis cuantitativo del paisaje. Índices ponderados

\begin{tabular}{|c|c|c|c|c|c|}
\hline Î́ndices & $4 \mathbf{4}_{1}$ & $\mathbf{4}$ & $55_{1}$ & 6 & 7 \\
\hline María Concepción & 0,25 & 0,72 & 1112,3 & 94,82 & 35,08 \\
\hline La Victoria & 3,24 & 5,22 & 60,1 & 59,93 & 54,35 \\
\hline Orsetti, Cerro Verde, Aguaí, Leiva, Bobby & 4,99 & 7,6 & 140,4 & 68,39 & 57,41 \\
\hline Roberts & 2,01 & 4,49 & 144,8 & 33,83 & 44,83 \\
\hline San Patricio & 3,37 & 5,5 & 56 & 64,76 & 54,09 \\
\hline Yuyo, Singer & 2,78 & 4,84 & 67,5 & 38,47 & 43,30 \\
\hline Yohasá 1 & 2,89 & 5,24 & 210,7 & 54,73 & 55,81 \\
\hline Yohasá 2, 3, 4 & 3,69 & 6,53 & 119,7 & 51,95 & 48,77 \\
\hline Yohasá 5 & 7,5 & 11,37 & 81 & 50,99 & 65,14 \\
\hline Yohasá Viejo & 8,08 & 12,42 & 57,1 & 44,20 & 44,8 \\
\hline Tres Capones & 2,97 & 5,72 & 185,1 & 59,70 & 57,02 \\
\hline López, Calzada de Piedra & 4,1 & 6,28 & 152,1 & 46,60 & 59,63 \\
\hline Tubomet & 3,92 & 7,1 & 221,4 & 51,05 & 49,61 \\
\hline San Justo & 4,22 & 6,87 & 96 & 71,37 & 51,34 \\
\hline El Playado & 4,20 & 6,81 & 79,3 & 44,23 & 60,94 \\
\hline El Carmelo & 4,41 & 5,91 & 113 & 16,39 & 25,31 \\
\hline Las Marías & 7,08 & 13,11 & 252,8 & 49,7 & 57,75 \\
\hline La Merced (Las Marías) & 4,27 & 7,31 & 172,2 & 63,46 & 49,13 \\
\hline La Merced (Topané) & 4,33 & 6,78 & 34,1 & 42,48 & 45,89 \\
\hline Garruchos & 2,35 & 4,10 & 73,1 & 31,39 & 21,31 \\
\hline La Península & 2,51 & 3,99 & 38,5 & 59,59 & 44,94 \\
\hline
\end{tabular}

\section{CONCLUSIONES}

Lo expresado plantea que los valores de alfa y de beta diversidad para la flora y la fauna, dependerán en gran medida de un factor de contexto. U na muestra del mismo tamaño, tomada en un pinar de la misma especie, plantada en igual período y con el mismo tratamiento silvícola, podría tener diferente diversidad en tanto se encuentre en uno u otro patrón de paisaje. Estos índices permiten un control más real y eficaz de los cambios en la biodiversidad de los componentes del paisaje (especialmente de flora y defauna). 
$\mathrm{H}$ ay consenso generalizado en que el avance de las actividades humanas sobre el paisaje nativo (agricultura, ganadería, forestaciones, urbanizaciones, etc), produce reducción del área total del hábitat disponible para la vegetación y la fauna nativas, determina el aislamiento de las parcelas y, la falta de conectividad, acrecienta la exclusión de muchas especies que viven en el paisaje nativo (M cGarigal y Marks, 1995; M iller, 1996, Solbrig, 1991).

L a disminución drástica del tamaño de los parches, asociada a un excesivo aumento de la distancia entre parches, puede producir reducción en la capacidad compensadora del microclima de los parches, caída de la riqueza de especies y de bioformas, afectando principalmente a la fauna de invertebrados (D evinski y Holt, 2000), pudiendo impactar negativamente sobre la oferta de alimentos para anfibios, reptiles, aves y mamíferos.

A pesar de esto los estudios cuantitativos sobre la fragmentación del paisaje y sus efectos sobre la biodiversidad, son aún incipientes y al gunas investigaciones dan cuenta de mayor diversidad en sitios forestados (B arret y Tressens, 1996).

Las hipótesis que expresan la retracción o segregación de algunas especies y la posibilidad de invasión por especies exóticas, en la medida que disminuye el tamaño de las células (lomas) del paisaje (O'N eill et al., 1999, Solbrig, 1991), probablemente resulten válidas para los paisajes prístinos, pero no siempre para los paisajes cultural es en los que se ha sustituido la matriz original del paisaje. En estos casos, el aumento de las parcelas (en calidad y variedad de tamaño) aumenta la heterogeneidad del paisaje conformando un patrón de mayor oferta de hábitat, con mayor ecodiversidad, con mayores recursos y mejores posibilidades de compensación de los factores de stress ambiental para muchos organismos. Lo expresado podría explicar la razón por la cual algunos paisajes con fuerte intervención antrópica (B arret y Tressens, 1996; este estudio) tienen alta riqueza de especies. Tal como plantea Solbrig (1991) la diversidad de un sistema puede ser consecuencia del número de individuos de distintas especies que entran y que salen del sistema (Hipótesis 3.8, del citado trabajo). En consecuencia, la mayor extensión de borde con parches de diferente composición específica y la mayor conectividad entre estos elementos, podría explicar la mayor diversidad en algunos sitios alterados por el hombre, en tanto el diseño de las parcelas favorezca el flujo entre los parches.

I gualmente importante resulta la geometría de la parcela y el contexto en que se encuentra comprendida. A sí por ejemplo, en este estudio, el predio Roberts, por ser una franja de campo, el borde expuesto (perímetro) es muy grande y la conectividad con los predios vecinos es muy alta. En este caso, hay fuertes contactos con pajonales y bosques de galería de la cuenca del $\mathrm{A} \cong \mathrm{G}$ arabí, con yerbales de baja densidad y pastizales en el límite sur y con paisajes mucho más heterogéneos en el límite norte del campo (incluyendo montes nativos, yerbales de baja densidad, talas rasas, parches pequeños de pastizales, áreas agropecuarias y de pajonales, entre otros usos). Estos intercambios poblacionales pueden determinar al ta riqueza de especies (UN NE-G L M , 2001).

$L$ a diversidad específica depende, en gran medida, de las transformaciones del paisaje y es por esto que son precisos estudios de ecodiversidad como base para la interpretación de los resultados producidos mediante la aplicación de índices de diversidad específica a nivel de uno o más predios.

En otro sentido, el paisaje y sus ensambles poblacionales son complejos que varían en el tiempo en el tiempo y en el espacio, por lo que una idea más real se obtiene 
de comparar los distintos estados que se dan en relación a la estacionalidad de la flora y de la fauna y a los cambios de manejo en determinado proyecto.

L os paisajes de planicies inundables de grandes ríos, están compuestos por células (manchas) de muy variado tamaño y, algunas, de sólo pocas hectáreas, respondiendo al patrón geomorfológico y al hidroperíodo (Malvárez, 1997; Casco, 2003; N eiff y $M$ alvárez, 2004). Esta condición natural o celularidad del sistema, no debe confundirse con la atomización de los paisajes fragmentados, en los ecosistemas terrestres, como consecuencia de la expansión de las actividades humanas. Con esta salvedad, puede señal arse que ambos paisajes comparten la característica de tener gran número de elementos, de distinta conectividad. Los índices que se presentan en esta contribución pueden ayudar en el mejor conocimiento de ambas situaciones proveyendo bases más objetivas para la evaluación de la biodiversidad en programas de control ambiental nivel de predios y a nivel regional.

En la planicie del A Ito Paraná, se ha explicado la estabilidad de la vegetación de los bañados, por la existencia de metapoblaciones (B ini et al. , 2001). El análisis de metapoblaciones (McCullough, 1999) requiere disponer de herramientas que permitan cuantificar los valores local es de un determinado ensamble biótico dentro de un contexto más amplio de paisaje, mediante índices como los que hemos presentado.

\section{BIBLIOGRAFÍA}

BARRet, W.H. y S.G. TRessens, 1996. Estudio de la vegetación nativa en plantaciones de Eucalyptus grandis (M yrtaceae) en el norte de la provincia de Corrientes, República A rgentina. Bonplandia, 9 (1-2): 1-18.

BINı, L.M., S.M. Thomaz y D. SouzA, 2001. Species richness and ?-diversity of aquatic macrophytes in the U pper Paraná River floodplain. Arch. Hydrobiol., 151 (3): 511-525.

Capurro, R.A. y R. Carnevali, 1985. Regiones Naturales de Corrientes. ID IA (309-10): 69-76.

CAsco, S.L., 2003. Poblaciones vegetales centrales y su variabilidad espacio-temporal en una sección del Bajo Paraná influenciada por el régimen de pulsos. Tesis D octoral. U niversidad N acional del N ordeste. Corrientes, $127 \mathrm{p}$.

DEVINSKI, D.M . y R.D. HOLT, 2000. A survey and overview of habitat fragmentation experiments. Conservation Biology, 14 (2): 342-355.

Etchechuri, H., R. Ferraro y G. BengoA, 2002. Evaluación de Impacto A mbiental. Entre el saber y la práctica. Centro de Investigaciones A mbientales (CIAM ). Espacio, Buenos A ires.

Federal Environmental A ssessment Review Office (fearo). 1978. Ecological land survey guidelines for environmental impact analysis. Ecological L and Classification Series. Federal Environmental A ssessment and R eview Process. 13: 42 p. L ands Directorate Environment Canadá.

HAWKSWORTH, D.L. (ed.), 1995. Biodiversity. Measurement and estimation. Chapman \& Hall. The Royal Society, Oxford, $140 \mathrm{p}$.

HUSTON, M.A., 1999. Local processes and regional patterns: apropiate scales for understanding variation in the diversity of plants and animals. Oikos, 86 (3): 393-401.

M ALVÁREZ, A.I., 1997. Las comunidades vegetales del Delta del río Paraná. Su relación con factores ambientales y patrones del paisaje. Tesis doctoral. Universidad Buenos A ires, Buenos A ires, 167 p.

M CCullough, D.R. (ed.), 1999. M etapopulations and wildlife conservation. Island Press, Washington, 5384. 
MCGARIGAL, K. y B. MARKS, 1995. FRAGSTATS: Spatial pattern analysis program for quantifying landscape structure. Gen. Tech. Rep. PNW-GTR-351. Portland, OR: U.S. Department of A griculture, Forest Service, Pacific N orthwest R esearch Station. 122 p.

M ILLER, K.R., 1996. En busca de un nuevo equilibrio. World Resources Inst. 1-80.

NAVEH, Z. 1994. From Biodiversity to Ecodiversity: a Landscape-E cology approach to Conservation and Restoration. Restoration E cology, 2 (3): 180-189.

NeIfF, J.J ., 2001. Diversity in some tropical wetland systems of South A merica. Pp. 1-60. En: W. Gopal, J. Junk y J.A. Davis (eds.). Biodiversity in wetlands: assessment, function and conservation. V 2. Backhuys Publishers, Leiden, The N etherlands.

NeIfF, J.J y A.I. M A LVÁrez, 2004. Grandes Humedales Fluviales. Pp. 77-85. En: A.I. M alvárez y R.F. Bó (compiladores). D ocumentos del curso-taller Bases ecológicas para la clasificación e inventario de humedales en Argentina FCEN (UBA) - RA M SA R - USFW S - USDS, Buenos Aires.

NeIFF, J.J ., S.L. CASCO y J.C. A RIA S, 2003. Glosario de humedales latinoamericanos. Pp. 336-376. En: J.J. $\mathrm{N}$ eiff (ed.). Humedales de Iberoamérica. CY TED, Programa Iberoamericano de Ciencia y Tecnología para el Desarrollo-Subprograma X VII. Red Iberoamericana de Humedales, Cuba.

O'NeILL, R.V., K.H. RITTERS, J.D. WICKHAM y B. JONES, 1999. L andscape pattern metrics and regional assessment. E cosystem health, 5 (4): 224-231.

PICKET, S.T.A. y P.S. WhITE, 1985. The ecology of natural disturbance and patch dynamics. N ew York A cad. Press, N ew Y ork, $456 \mathrm{p}$.

SolBRIG, O., 1991. Biodiversity. Scientific issues and collaborative research proposals. M A B Digest 9. UNESCO, París: 77 p.

TN C (The N ature Conservancy), 2000. Diseño de una G eografía de la Esperanza. V ol. 1 y 2. 10 capítulos y 28 apéndices.

U NN E-GLM (U niversidad Nacional del Nordeste-Grupo Las Marías), 2001. Evaluación de Impacto Ambiental. Tomos 1 y 2.5 capítulos.

WIENS, J.A., 1996. Wildlife in patchy environments: metapopulations, mosaics, and management. En: D.R. McCullough (ed.), M etapopulations and wildlife conservation. Island Press, W ashington, pp 53-84.

WRI-UICN/PNUM A/Instituto de Recursos M undiales, 1992. Estrategia Global para la Biodiversidad. 1243.

Zunino, G., 1986. Algunos aspectos de la ecología y etología del mono aullador negro (Alouatta caraya) en hábitat fragmentados. Tesis doctoral. U niversidad de Buenos A ires, B uenos A ires, $152 \mathrm{p}$.

Recibido/Received/: 18-Feb-05

A ceptado/A ccepted/: 07-J ul-06 